\title{
NOTICE HORPHOLOGIQUE
}

\section{SUR LES \\ GRYLLOTALPIENS}

PAR

\section{H. DE SAUSSURE et L. ZEHNTNER}

Avec les Planches XVI et XVII.

La tribu des Gryllotalpiens forme, dans la famille des Grillides, et même parmi les Orthoptères sauteurs, un groupe en apparence très nettement séparé.

On a jusqu'à ce jour basé ce groupe sur les caractères suivants :

Pattes antérieures fouisseuses, ayant leurs tibias transformés en instrument aratoire. Femelles dépourvues d'oviscapte.

Le fait de posséder des pattes fouisseuses, de même que les habitudes souterraines de ces insectes, rattachent les Gryllotalpiens péremptoirement à la famille des Gryllides et particulièrement à la tribu des Grylliens.

Quant à l'absence d'oviscapte, elle constitue, parmi les Orthoptères sauteurs, une exception unique. Comme l'oviscapte est formé aux dépens des deux derniers segments de l'abdomen, son absence entraîne une différence très frappante dans la composition et le nombre apparents des pièces de cette partie du corps. Les derniers segments ventraux n'étant pas modifiés 
chez les femelles pour la formation de l'oviscapte, l'abdomen dans ce sexe ne diffère pas essentiellement dans sa composition de celui des mâles.

L'absence d'oviscapte est, comme on le comprend, une couséquence du genre de vie souterrain. Des insectes vivant dans des galeries et creusant des excavations pour y construire leurs nids n'ont besoin d'aucun instrument perforant pour y déposer leurs œufs.

Les pièces anales des Gryllotalpiens offrent du reste, d'un genre à l'autre, des différences si singulières que leur analyse présente un intérêt particulier, et comme elle n'a jamais été faite d'une manière complète, nous avons été conduits, en nous occupant de ce groupe d'insectes, à lui accorder quelque attention.

Disons sans plus tarder que la diagnose ci-dessus donnée de la tribu des Gryllotalpiens n'est pas exacte, attendu que dans l'un des genres qui la compose, on trouve chez les femelles un oviscapte parfaitement caractérisé.

L'abdomen des Orthoptères doit être considéré comme composé de 10 segments complets, en comptant comme tels :

$1^{\circ}$ Le segment de sa base, désigné sous le nom de médiaire, parce qu'il établit la liaison entre le thorax et l'abdomen, et qu'il participe en partie encore aux caractères du thorax.

$2^{\circ}$ L'anus formé d'une plaque tergale et de deux plaques ou valvules ventrales ${ }^{1}$.

${ }^{1}$ Comme dans les descriptions d'espèces on ne compte généralement pas le segment médiaire, vu les apparences diverses qu'il revêt, il est essentiel de dire que dans l'analyse qui suit, nous le comptons toujours comme formant le ler segment de l'abdomen, d'où résulte que l'anus devient le $10 \mathrm{me}$ segment.

Pour plus de brièveté, et pour tout ce qui concerne la composition de l'abdomen dans les Orthoptères, nous renvoyons à l'excellent mémoire de M. CH. Brunner de Wattenwyl: Die morpholoyische Bedeutung der Segmente, speciell des Hinterleibes, bei den Orthopteren, Wien, 1876, en adoptant la même numérotation pour les tergites et les ventrites de l'abdomen, ce qui nous dispense d'entrer ici dans de longs détails sur la composition typique de cette partie du corps. 
Les deux appendices apicaux qui se retrouvent dans tous les Orthoptères, soit les cerci, appartiennent au $10^{\mathrm{me}}$ segment et doivent être considérés comme étant une expansion de la membrane pleurale de ce segment. M. BRunner de WattenwyL suppose qu'ils pourraient être le résultat d'un développement des stigmates de ce segment. Quoi qu'il en soit, il faut les considérer comme étant des appendices latéraux et non une dépendance des valvules de l'anus. Ces appendices, souvent composés d'une seule pièce, ont une tendance à se segmenter en articles plus ou moins nombreux et plus ou moins bien séparés. Chez les mâles, ils prennent souvent des formes très accentuées et jouent alors un rôle positif dans l'acte de l'accouplement.

Les Gryllotalpiens se divisent comme suit au point de vue de leurs pièces anales :

1.) L'abdomen ordinaire, n'offrant d'autres appendices que les cerci. Femelles sans trace d'oviscapte. Mâles offrant un pénis charnu renforcé de pièces cornées. Gryllotalpites Sauss. 1,1.) L'abdomen portant, outre les cerci, deux appendices anaux placés en dessous des premiers. Le pénis chez les mâles non apparent (rétractyle?). Tridactylites Sauss.

2.) Femelles dépourvues d'oviscapte. G. tridactylus Latr.

'2,2.) Femelles munies d'un oviscapte corné très court. G. Rhipipteryx Newm.

\section{GRYLLOTALPITES}

Dans ce groupe, l'abdomen a, chez les deux sexes, la même composition apparente, offrant 10 tergites et 9 ventrites distincts.

L'anus est formé par les trois valvules habituelles (fig. 1, 2, $8,9, v s, v i)$, coriacées extérieurement, charnues intérieurement. La valvule supérieure $v s$ (soit la plaque suranale) est le résultat d'un prolongement médian du dernier tergite (z), et les deux 
valvules inférieures $(v i)$ représentent le dernier $\left(10^{\mathrm{me}}\right)$ segment ventral.

Le dernier tergite ( $z$ ) est en apparence court et transversal, recouvrant la base des cerci $(c)$ par ses côtés, et la valvule supérieure de l'anus ( $v s)$ semble souvent former un segment séparé (fig. 8), mais il n'en est rien : cette valvule appartient en réalité au dernier tergite, et constitue, avec la partie basiliaire de ce segment, un seul tout; elle n'en est séparée que par une sorte de gouttière transversale.

Les valvules anales inférieures $(v i)$ représentent, avons-nous $\mathrm{vu}$, le $10^{\mathrm{me}}$ segment ventral divisé en deux parties. Au-dessous de ces valvules, on peut encore distinguer une sorte de lame membraneuse transversale (fig. $9, m$ ) qu'il faut peut-être considérer comme une dépendance de la membrane d'union entre les $9^{\mathrm{me}}$ et $10^{\mathrm{me}}$ ventrites, et qu'on pourrait au besoin envisager comme étant l'arceau correspondant à la pièce basiliaire $(z)$ du $10^{\text {me }}$ tergite $^{1}$.

A sa face ventrale, l'abdomen offre entre les deux sexes une légère différence.

Chez les femelles: (fig. 1), le $8^{\text {me }}$ ventrite est rétréci, arrondi en arrière. Le $9^{\text {me }}$ est transversal, court, souvent rétréci et apparent seulement de chaque côté, recouvert au milieu par le $8^{\mathrm{me}}$. Dans les nymphes et dans les larves, le $9^{\mathrm{me}}$ segment est plus grand, saillant et très apparent; c'est seulement chez les femelles adultes qu'il se raccourcit, avec tendance à s'invaginer, sans doute pour faciliter l'acte de l'accouplement.

Chez les mâles: (fig. 2), les ventrites sont tous très apparents. Le $9^{\mathrm{me}}$ est court et transversal ${ }^{2}$.

${ }^{1}$ Lacaze-Duthiers (Ann. des Sc.nat., t. XVII, 185̈2, p. 208, pl. 10 à 12) compte à l'abdomen 11 segments. Pour luí, l'anus forme le 11 me segment, composé des trois valvules anales. L'auteur sépare la valvule dorsale du 10 me tergite; la lame membraneuse ventrale invaginée représenterait le ventrite du $10 \mathrm{me}$ seg ment. Cette manière de compter ne nous semble guère admissible en pratique, car la plaque suranale ne forme qu'un seul tout avec le $10 \mathrm{me}$ tergite.

${ }^{2}$ H. de Saussure, Mélanges orthoptérologiques, 5’e fasc., pl. 11, fig. I, 6-8. La figure 7 (우) n’est pas exacte, le $9 \mathrm{me}$ ventrite n’ayant pas été représenté. 
Le pénis (fig. 3), placé sous l'anus et recouvert en dessous par la plaque sous-génitale ( $9^{\mathrm{me}}$ ventrite), est formé par une masse charnue arrondie $(p)$, parcourue en dessus par une gouttière $(g)$, servant probablement à conduire la liqueur séminale et offrant à sa base une dépression $(f)$ L'organe est renforcé par des pièces cornées dont l'ensemble se rapproche de la forme d'une ancre (fig. 3, brs; 4, 5), comme l'a indiqué M. BRunner DE WATtenwyl (l. c., p. 11), mais de configuration variable suivant les espèces.

Cet appareil se complète par deux autres pièces cornées qu'il faut considérer comme étant les homologues des titillatores (Brunner) ou crochets, si apparents chez les Blattides, Mantides et autres Orthoptères, mais qui, chez les Gryllotalpiens, ne se se révèlent que par la dissection des pièce génitales.

Les pièces cornées du pénis revêtent, comme il vient d'être dit, dans chaque espèce des formes particulières.

Gryllotalpa vulgaris. Dans cette espèce, l'ancre (fig. 4, 5) se compose d'une branche médiane aplatie (ai) et d'un arc transverse $(b)$ placé en dessus et un peu de champ, et soudée à la branche médiane. Les deux extrémités de l'arc se terminent par un élargissement bifurqué, qui se présente sous des apparences très différentes, suivant le côté par lequel on les regarde. Son apophyse antérieure (fig. 4, 5, s) est un peu crochue au bout; la postérieure $(r)$, vue par sa face supérieure (fig. 4), apparaît comme aplatie et arrondie à son extrémité; vue de profil (fig. 5) elle est comme subulée. L'arc en place, vu en dessus, dans son ensemble (fig. 3), apparaît sous la forme d'une couronne à deux dents $b$ (comp. L. H. Fischer, Orthop. Europ., tab. III, fig. 4). La branche longitudinale est large et déprimée. En arrière, elle dépasse un peu la branche transversale (fig. 3, 4, 5, i); à son extrémité antérieure $a$ elle est dilatée de chaque côté. En dessous (fig. 5), elle est plate et même un peu concave. Vue in situ, la branche longitudinale (fig. $3, i$ ) paraît fort courte, parce que sa partie moyenne et antérieure $o$ est vue en raccourci. 
L'ancre tout entière est mobile et, suivant les contractions du pénis, sa branche médiane (o) peut s'avancer jusqu'à l'extrémité des dépressions $f$ (fig. 3 ). Ce mouvement de l'ancre joue probablement un rôle dans l'acte de l'accouplement et dans la fécondation de la femelle.

Les titillateurs forment deux pièces cornées libres, engagées au-dessous de l'ancre et la débordant en avant (fig. $3, t$ ). Ils se composent chacun d'une lame tordue dont l'extrémité est élargie et courbée en forme de spatule (fig. $6,7, c$ ), tandis que leur partie basilaire $(e)$ est dilatée en dessus en forme de triangle (fig. 7) et se termine en avant par un prolongement spiniforme. Ces organes sont mobiles, pouvant glisser d'avant en arrière, il est possible que, dans l'accouplement, leur épine terminale atteigne la vulve de la femelle.

Gr. africana. La branche transverse de l'ancre (fig. 10, $11, b)$ est doublement arquée, et la branche longitudinale (o) est plus étroite. A sa face inférieure, celle-ci semble être percée (fig. 11); à sa face supérieure, elle offre deux corps blanchâtres charnus (fig. 10,v) d'une apparence glandulaire. Cette branche repose dans une dépression triangulaire du pénis, qui se termine par deux replis en sillons disposés en forme de $\mathrm{V}$. Les branches latérales de l'arc transversal sont grêles, fortement recourbées en dessous (fig. 12) et terminées d'une manière arrondie et à peine dilatée. Les titillateurs (fig. 13) sont soudés en une seule pièce. Leurs ailerons latéraux $(c)$, tordus comme chez le Gr. vulgaris, sont excavés en forme de cuillère et recourbés en haut, convexes en dessous (fig. 14, branche gauche).

Les Scapteriscus ne semblent pas offrir dans le pénis de différences génériques. Chez le Sc. didactylus L. (fig. 15), l'arc de l'ancre et la branche longitudinale sont partagés par une ligne membraneuse longitudinale, et les extrémités latérales de l'arc sont divisées en deux cornes à peu près comme chez la Gryllo- 
talpa vulgaris. Les deux titillateurs (fig. 16) sont indépendants l'un de l'autre comme chez cette espèce.

Sous le rapport de l'armature du pénis, les Scapteriscus diffèrent bien moins de la Gryllotalpa vulgaris que la Grillotalpa africana ne diffère de cette dernière, aussi peut-on envisager le genre Scapteriscus moins comme un genre naturel que comme un genre de convenance.

Genre Cylindrodes Gray. Ce genre, encore mal connu, offre des formes aberrentes. L'abdomen est très long, grêle et cylindrique, mais ses pièces anales ne semblent différer de celles des Gryllotalpa que par. leurs proportions. Nous ne pouvons malheureusement les décrire que d'après un seul individu, en fort mauvais état, dont le sexe reste incertain, mais que nous supposons être une femelle.

L'extrémité de l'abdomen (fig. 17-19) est à peine rétrécie (sur la figure donnée par GRAY elle est même plutôt renflée). Le $9^{\mathrm{m} \theta}$ segment dorsal (fig. 17) est très long, convexe, faiblement arrondi au bout. Le $10^{\mathrm{m} \theta}$ dépasse à peine le $9^{\mathrm{me}}$ et n'apparaît que sous la forme d'une étroite bande transversale presque linéaire (fig, 18, $z$ ), mais il se prolonge au milieu comme d'habitude pour former la plaque suranale. Celle-ci est rabattue en bas dans notre individu desséché. Les cerci sont très courts, ovalo-coniques, non divisés en articles. Le $9^{\mathrm{me}}$ segment ventral (fig. 18) est arrondi en forme de dé à coudre. Après l'avoir enlevé, nous avons trouvé en arrière des valvules anales une sorte de bourrelet charnu (fig. 19, b) dont les angles se prolongent en arrière sous la forme de deux dents coriacées. C'est peut-être là le pénis ; mais cette masse desséchée est trop déformée pour qu'il nous soit permis de rien affirmer de positif à cet égard. Nous n'avons trouvé ni ancre, ni titillateurs, ce qui nous a fait supposer que notre individu est une femelle. 


\section{TRIDACTYLITES}

Ces insectes forment un groupe entièrement séparé. Seuls parmi les Orthoptères, ils possèdent dans les deux sexes 4 appendices postérieurs.

Les deux appendices surnuméraires (fig. 21-24, a) sont placés au-dessous des cerci et leur ressemblent, étant de forme allongée, cylindrique ou subcomprimée, et étant ciliés comme les cerci. L'homologie de ces appendices n'a point encore été

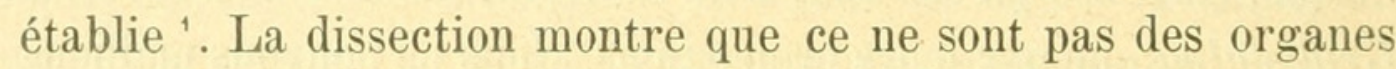
spéciaux, mais de simples processus des valvules inférieures de l'anus (fig. 23, a).

Les fonctions de ces appendices sont difficilement explicables; elles semblent toutefois être en rapport avec les mœurs aquatiques des Tridactyliens, puisque ces organes ne se retrouvent pas chez les autres Orthoptères. Peut-être servent-ils à appuyer à la surface de l'eau pour soutenir la partie postérieure du corps. Les pattes postérieures n'étant organisées que pour le saut et pour la propulsion, et n'offrant pas comme les autres pattes un tarse capable de se poser à plat sur la surface liquide, mais se terminant par des éperons aigus, ne peuvent en effet guère remplir que dans l'eau les fonctions de rames. Il est probable aussi que les deux appendices surnuméraires combinés avec les cerci servent, grâce à leurs poils, à retenir une certaine quantité d'air autour du corps, comme le font aussi les fémurs, lorsque ces insectes sont submergés.

L'abdomen des Tridactyliens offre tantôt la même composition que celui des Gryllotalpiens, tantôt une composition différente en

\footnotetext{
${ }^{1}$ On a lieu d'être surpris qu'aucun auteur n'ait cherché à élucider la signification de ces appendices. Ni L. Dufour, ni Foudras, qui ont cependant trouvé en abondance des Tridactylus vivants, ne se sont occupés de ces appendices. L. - H. Fischer n'a pu y réussir, n'ayant possédé, à ce qu'il paraît, qu'un ou deux individus desséchés de chaque sexe.
} 
ce que, chez les femelles, les deux derniers segments ventraux sont transformés et forment un oviscapte.

Le $10^{\mathrm{me}}$ tergite est quelque peu différent suivant les sexes.

Chez les femelles (fig. 21, 25, 31), il se divise en trois parties; deux latérales $(l)$, représentant le $10^{\mathrm{me}}$ tergite proprement dit (fig. 8, 21, z) et une médiane (ou plaque suranale), prolongée entre ces dernières, plus ou moins cordiforme, lorsqu'elle est dépliée (fig. 31,vs), étroite, creusée en gouttière, comprimée, et à bords relevés, lorsqu'elle est dans sa position naturelle (fig. 21, 25). Cette partie médiane est la seule apparente en dessus, les parties latérales étant en général recouvertes par le segment précédent.

Chez les mâles (fig. 24, 28), la partie médiane, ou plaque suranale $(v s)$ est en général plus large, plus carrée et moins ployée. Le pénis n'est pas apparent, étant probablement entièrement rétractile, et ne semble pas porter d'ancre ni de titillateurs.

\section{Genre Tridactylus.}

Femelles. Les deux derniers segments ventraux ont une tendance à se fendre longitudinalement dans le but manifeste de former un oviscapte (fig. 20). Le $8^{\text {me }}$ segment, qui correspond à la plaque sous-génitale $@$ des Orthoptères munis d'un oviscapte, offre, au milieu de son bord postérieur, une petite échancrure; de même que la plaque sous-génitale des Locustides.

Ce segment est suivi d'une dépendance ou plaque cornée $(u)$ divisée en deux moitiés et formant comme deux valves déployées. Il est probable qu'en se rapprochant pour former un angle dièdre, ces deux valves servent à conduire les œufs, remplissant les fonctions d'un oviscapte. Dans sa position habituelle, cette pièce fendue n'est guère visible, étant presque entièrement recouverte par le $8^{\mathrm{me}}$ ventrite. Elle est incontestablement l'homologue des deux valves inférieures de l'oviscapte des 
Orthoptères sauteurs et doit être considérée suivant le système de M. Brunner DE Wattenwyl, comme formé par la membrane du $8^{\text {me }}$ segment ventral.

Le dernier ou $9^{\text {me }}$ segment ventral apparent est plus ou moins arrondi. Il est divisé par un sillon longitudinal indiquant une tendance à se partager en deux moitiés, qu'il faut considérer comme étant les homologues des valves supérieures de l'oviscapte ${ }^{1}$.

Mâles. L'apparence extérieure des derniers segments ventraux est sensiblement la même que chez les femelles, mais avec cette différence que l'annexe du $8^{\text {me }}$ segment n'existe pas et que le bord postérieur de ce segment n'est pas échancré. Enfin, le $9^{\text {me }}$ segment n'est pas partagé par un sillon longitudinal. Ce n'est guère qu'à l'absence de ce sillon et à l'absence de l'échancrure du $8^{\text {me }}$ segment que l'on peut distinguer les mâles des femelles, car l'annexe du $8^{\text {me }}$ segment $@$ est en général, comme il a été dit, invaginé et peu ou pas apparent.

Dans les deux sexes, le $10^{\text {me }}$ tergite ou segment anal, est corné dans ses parties antérieures et latérales et sa partie cornée se divise en plaques diverses (fig. 24).

Les valvules inférieures dè l'anus (fig. 23, vi) restent seules membraneuses, mais elles ont leur bord latéral chitinisé.

Les cerci (fig. 21-24,c) ne sont composés que de deux articles et les appendices anaux $(a)$ n'offrent aucune trace de division.

${ }^{1}$ L.-H. Fischer a figuré presque exactement l'abdomen $q$ des Tridactylus. Il signale la différence qui règne entre les sexes dans la forme du 8 me segment ventral. Il semble avoir distingué l'annexe de ce segment chez les femelles, mais sans avoir remarqué que cette pièce est partagée en deux moitiés. En revanche, il figure le $9 \mathrm{me}$ ventrite comme s'il était réellement divisé en deux parties separées. Fieber accorde un oviscapte aux Tridactylus (Xya) 우. Il faut supposer qu'il a eu sous les yeux un Rhipipteryx, et que, ne connaissant pas ce genre, il l'a pris pour un Tridactylus. 


\section{Genre RHIPIPTERYX.}

Dans ce genre, les femelles possèdent un véritable oviscapte corné, très court, il est vrai, et qui rappelle celui des Acridides.

Femelles. Le $10^{\text {me }}$ tergite est divisé en trois parties, comme chez les Tridactylus (fig. 25, 31). Les deux parties latérales (fig. $31, l$ ) sont très apparentes. La partie médiane (vs), ou plaque suranale, lorsqu'elle est dépliée (fig. 31) est large et cordiforme, mais dans sa position habituelle (fig. 25) elle est comprimée, ployée, à bords relevés, se présentant sous la forme d'une raquette excavée au milieu. La base de cette partie, d'habitude recouverte par les pièces latérales, porte des plaques cornées (fig, 31). Les cerci (c) sont faiblement articulés (composés de 9 articles, plutôt indiqués que séparés) et la base de ces organes est renforcée par des pièces cornées (fig. 31). Les deux appendices $(a)$ sont assez gros et très obtus: chez les femelles, ils sont parfois comme partagés au milieu, formant deux articles imparfaitement séparés (fig. 31, R. mexicana Sss.) L'extrémité du tube intestinal (i) passe sous la base du $10^{\mathrm{me}} \mathrm{seg-}$ ment pour aboutir à l'anus. Il est recouvert en dessus par un arc corné non adhérent (fig. $31, r$ ), qui tient aux pièces latérales du $10^{\text {me }}$ tergite. En dessous (fig. 32), le tube intestinal est fermé par une membrane qui le réunit aux valvules anales et ne laisse apparaître que l'extrémité de la valvule supérieure.

A la face ventrale de l'abdomen, on ne trouve que 8 segments, mais ils sont suivis d'un oviscapte corné, très court (fig. 26, 27), formé :

$1^{\circ}$ De deux valves inférieures (fig. 26, 27, u), échancrées en dessous et terminées par une pointe grêle, souvent recourbée en bas suivant les espèces.

$2^{\circ}$ De deux valves supérieures formées par le $9^{\text {me }}$ segment, 
et qui sont certainement les homologues de la $9^{\text {me }}$ plaque ventrale des Tridactylus, tandis que les valves inférieures sont les homologues de la pièce fendue annexe du $8^{\text {me }}$ ventrite que l'on trouve chez ces derniers (fig. 20, $u$ ). Le $8^{\text {me }}$ segment ventral proprement dit (fig. 26) porte au milieu une petite échancrure. comme chez les Tridactylus.

Les valves intermédiaires de l'oviscapte manquent ou n'existent qu'à l'état rudimentaire.

Mâles (fig. 28-30). Chez les mâles, le $9^{\text {me }}$ segment dorsal est peu apparent, souvent seulement par ses angles (fig. 28); parfois tout à fait invaginé ( $R h$. mexicana). Le $10^{\text {me }}$ segment, ou segment anal, a ses côtés chitinisés. La plaque suranale (fig. 28,vs) est étroite et ployée ou faiblement cannelée (fig. 35). Elle porte de chaque côté un appendice lamellaire (fig. 28) allongé et densément strié en travers, de même longueur que la plaque et qui paraît être articulé à sa base. Ces appendices, qui sont probablement le résultat d'une segmentation de la plaque suranale semblent pouvoir s'écarter et se placer transversalement, recouvrant par leur extrémité la base des cerci (fig. 35). Les deux appendices anaux (a) ne sont pas divisés (comme chez les femelles de certaines espèces, fig. 31); ils sont larges et comprimés (fig. 30) et de forme variable suivant les espèces. Les valvules inférieures de l'anus (fig. 33, vi) sont assez longues; elles offrent, comme chez les femelles, des plaques cornées (fig. 28, 34, *). Dans la Rh. mexicana ơ (fig. 34) la dernière de ces plaques, occupant le bord postérieur des valvules inférieures de l'anus, forme un crochet $\left(^{*}\right)$ qui semble être libre et mobile, et qui imite les titillateurs de divers Orthoptères sauteurs, mais ces crochets n'appartiennent point au pénis et sont ici purement adventifs. Le $9^{\text {me }}$ segment ventral, ou plaque sous-génitale, est débordant ou arrondi (fig. 28-30, 35-37, 9i).

Les pièces anales sont du reste chez les mâles, et suivant les espèces, très variable dans leurs formes et dans leurs proportions. 
Ainsi chez le Rh. cyanipennis (fig. 35, 36), l'extrémité de l'abdomen est fortement dilatée comme chez les mâles de certains Acridiens (Caloptenus), quoique d'une manière différente en ce qui concerne la forme des derniers segments.

Chez cette espèce les cerci $(c)$ et les appendices anaux $(a)$ sont très courts : ces derniers un peu arqués; les lanières latérales de la plaque suranale sont étroites et transversales, et la base du $10^{\text {me }}$ segment dorsal est fortement relevée et extraordinairement dilatée en forme de conche cornée, formant de chaque côté un processus comprimé qui dépasse énormément l'abdomen en largeur. (Il est toutefois à supposer que l'individu figuré a ses pièces génitales déployées, ayant probablement été pris in copula.) En dessus, le $6^{\text {me }}$ segment est très court et recouvre les $7^{\text {me }}$ et $8^{\text {me }}$ tergites (fig. 36 ).

L'étude des nombreuses espèces de ce genre révélerait encore d'autres modifications. Ainsi chez la Rh. Brullei Sss. les appendices anaux sont comprimés et dilatés en massue; chez la $R h$. forceps Sss. ils sont arqués et dilatés au bout de manière à former ensemble une tenaille (fig. 37), etc.

Obs. 1. Entre les mâles et les femelles, il règne quelquefois aussi de grandes différences dans la tête, le chaperon et l'écusson facial des mâles offrant des rugosités singulières qui manquent chez les femelles (Rh. mexicana Sss.).

Il règne également des différences entre les deux sexes dans la forme des appendices anaux inférieurs.

Obs. 2. On a considéré les Tridactyliens comme ayant leurs tarses antérieurs et intermédiaires composés tantôt de 3 , tantôt de 2 articles seulement. Un examen exact de toutes les espèces à nous connues a montré que ces organes ne possèdent jamais que deux articles '.

${ }^{1}$ Le premier article de ces tarses, étant échancré en dessous, offre souvent à la loupe une apparence trompeuse qui peut faire croire a l'existence de 3 articles; cette apparence disparaît au microscope. Il est surprenant que SAvigny, 
CONSIDÉRATIONS GÉNÉRALES.

La tribu des Gryllotalpiens ne pouvant plụs être caractérisée par le fait que l'abdomen serait dépourvu d'oviscapte chez les femelles, on est fort embarrassé pour en donner une diagnose. En effet en dehors de ce caractère, on n'en trouve plus aucun autre qui puisse s'appliquer à tous les genres.

Les Gryllotalpiens ne forment pas, en effet, un groupe compact, mais plutôt une série par enchaînement.

C'est ce qui ressort de l'analyse suivante :

\section{Gryllotalpites.}

Ce groupe est caractérisé par l'absence complète d'oviscapte chez les femelles et par la forme des tibias antérieurs, qui sont courts, très fortement dilatés et convertis en une main aratoire, et avec le métatarse dilaté.

L'absence complète d'oviscapte est un fait absolument unique parmi les Orthoptères proprement dits, tous les autres possédant au moins les rudiments de cet organe, même lorsqu'il n'est pas apparent.

Si l'on n'envisageait que les Gryllotalpa (sensu latiore: Gryllotalpa et Scapteriscus), on pourrait rendre la diagnose de ce groupe plus générale, et, par opposition avec les Tridactylites, on pourrait ajouter que les Gryllotalpites sont de vrais Gryllides, possédant tous les caractères de la famille: tarses composés de 3 articles, antennes longues et sétacées, organes du vol conformes à ceux des Grylliens. Mais le genre Cylindrodes vient décaractériser le groupe des Gryllotalpites par ses formes

toujours si exact jusque dans les moindres détails, ait figuré les tarses du Tridactylus Savignyi Sss. comme composé de 3 articles. Nous n'avons trouvé sous le microscope que deux articles aux tarses de cette espèce égyptienne, comme à ceux des autres espèces. 
aberrantes, qui semblent être en contradiction, même avec les caractères de la famille des Gryllides; les antennes des Cylindrodes étant filiformes, courtes et composées d'un petit nombre d'articles; les pattes étant extraordinairement courtes et leurs tarses ayant perdu un ou deux articles et ne portant qu'une seule griffe.

Ces anomalies ne sont toutefois que la conséquence d'une adaptation à des mœurs particulières, les Cylindrodes, vivant emprisonnés dans les galeries qu'ils se creusent dans les tiges des plantes. L'étroitesse d'un pareil genre de vie a conduit à une sorte de dégradation des caractères assez analogue à celle que détermine souvent le parasitisme, spécialement en ce qui concerne la brièveté des pattes et la présence d'une griffe unique aux tarses.

Les Cylindrodes sont donc des Gryllotalpites, mais aberrants, on pourrait presque dire dégradés. Ils rentrent du reste dans la diagnose typique ci-dessus donnée.

\section{Tridactylites.}

Les espèces de ce groupe offrent entre elles des caractères identiques: $1^{\circ}$ dans la présence de 4 appendices anaux. $2^{\circ}$ dans l'énorme modification de leurs pattes postérieures. $3^{\circ}$ dans le fait que les tarses ont perdu une partie de leurs articles. En effet, les tarses des deux premières paires n'offrent que deux articles ${ }^{1}$, et ceux de la $3^{\text {me }}$ paire sont réduits à un seul article, qui ne sert plus à rien pour la marche .

1 Cette circonstance tient sans doute à un fait d'adaptation à la vie souterraine, qui demande aux tarses de pouvoir s'effacer en se repliant dans une rainure du tibia pour laisser a ce dernier toute liberté d'action lorsqu'il doit servir d'instrument aratoire. Chez les Gryllotalpa, l'adaptalion se fait d'une autre manière en ce que le métatarse antérieur se dilate et complète l'instrument de labour formé par le tibia. Une pareille modification ne pourrait guère s'adapter à la vie aquatique des Tridactylites, car ce sont les tarses des deux premières paires qui servent à maintenir ces insectes à la surface des eaux.

${ }^{2}$ Le tibia appuie sur le sol par l'extrémité de ses deux longs éperons infe- 
Enfin, les Tridactylites offrent divers autres caractères communs, tels que la nature cornée des élytres; la structure en éventail des ailes, avec le champ marginal corné; enfin la brièveté et la structure presque monoliforme des antennes. Ce dernier caractère est de tous le moins important, car il se retrouve parmi les Gryllotalpites dans le genre Cylindrodes, comme il vient d'être dit.

A considérer leur genre de vie, les Tridactylites forment, sous ce point de vue aussi, un groupe bien séparé des Gryllotalpites, car ils sont surtout des insectes aquatiques, habitant le bord des rivières et des lacs, aimant à se mouvoir à la surface des eaux et ne craignant point d'être submergés '.

Les Tridactylus sont encore des insectes laboureurs; ils se rattachent aux Gryllotalpites par la forme du corps, qui est modelée en vue du travail souterrain. Leur pronotum ressemble beaucoup à celui des Gryllotalpa, quoique plus court et déjà échancré aux épaules; leur tête est encore assez conique, avec des yeux peu saillants, comme il convient pour percer le sol; les tibias antérieurs sont robustes, armés de fortes griffes et très propres à remuer la terre; enfin leur abdomen est dépourvu d'oviscapte chez les femelles ${ }^{2}$, comme chez les Gryllotalpites.

rieurs, qui sont un peu cannelés en truelle et légèrement crochus au bout, offrant ainsi un point d'appui très solide pour le saut. Le tarse réduit au métatarse, également un peu crochu au bout et n'appuyant également que par son extrémité, et seulement lorsque le tibia est replié en dessous, forme comme un ressort propulseur mobile, mais qui n'est ici qu'un organe auxiliaire, car chez diverses espèces il est entièrement atrophié et réduit à un simple tubercule.

${ }^{1}$ Les Tridactylites ne sont cependant pas les seuls Orthoptères affectionnant la vie aquatique. Parmi les Phasmides les Prisopus, et parmi les Mantides les Tarachodes paraissent aimer à s'immerger. Dans la famille des Tetticides, les Scelimena, observés par A. Humbert, ont des habitudes fort analogues à celles des Tridactylites. Ce ne sont pas des insectes fouisseurs, mais ils se jouent à la surface des eaux en s'aidant de leurs ailes brillantes et sautant sous l'eau lorsqu'ils sont submergés. A cet effet, leurs métatarses postérieurs sont dilatés en palettes. (Cp. H. DE Saussure, Annales ent. de Fr., IVme série, t. I, 1861 , p. 481 .)

${ }^{2}$ Quoique offrant déjà une tendance à le former. (Cp. p. 411.) 
Quant aux Rhipipteryx, ils s'écartent beaucoup plus que les Tridactylus des Gryllotalpites, au point que l'on peut se demander s'ils sont bien encore des insectes fouisseurs. En effet, leurs pattes antérieures sont assez longues; leurs tibias ne sont guère dilatés en palmette (bien que présentant encore un sillon dans lequel le tarse peut s'effacer), et l'extrémité n'est armée que de très courtes épines; leur tête n'est plus conique, mais presque perpendiculaire, comme chez les Acridides, et leurs yeux sont bombés; conditions toutes très peu favorables pour le travail souterrain. Enfin, la présence chez les femelles d'un oviscapte semble indiquer que ces insectes déposent leurs œufs à la manière des Acridides, en enfonçant dans le sol l'extrémité de leur abdomen, et que par conséquent ils n'occupent pas des chambres souterraines.

Il faut remarquer, aussi que le pronotum est plus court, plus en forme de selle, c'est-à-dire plus échancré aux épaules, à bord postérieur plus avancé que chez les Tridactylus, et que le reste du thorax est prismatique, un peu tronqué à son extrémité postérieure, ne formant pas un tout continu avec l'abdomen comme chez les Gryllotalpites et les Tridactylus. Ce sont là autant de caractères qui appartiennent à la vie aérienne. D'autre part, les Rhipipterix sont mieux ailés que les Tridactylus et leurs tibias postérieurs ne sont pas armés de palettes natatoires; leurs formes sont plus sveltes et plus légères aussi; d'où l'on peut conclure qu'ils ne sont pas aussi bons nageurs que les Tridactylus.

Il faut donc supposer que si les Rhipipteryx creusent, ils n'exécutent pas de grands travaux souterrains; que leur vie est surtout aérienne et qu'ils se meuvent soit sur le sol, soit à la surface des eaux; qu'ils évitent de s'enfoncer sous l'élément liquide en s'envolant, et que, lorsqu'ils sont submergés, c'est surtout grâce à la légèreté de leur corps, enveloppé de bulles d'air, qu'ils reviennent à la surface. 
Dans leurs affinités, ils s'écartent des Gryllotalpites pour se rapprocher plus particulièrement des Acridides, auxquels ils se relient par la présence et la forme de leur oviscapte. On a vu aussi que l'extrémité de l'abdomen des mâles est également sujette, comme chez certains Acridides, à prendre des formes exagérées et très singulières par suite de la tuméfaction des derniers segments (Rh. cyanipennis, p. 415).

En résumé de ce qui précède, on peut dire :

$1^{\circ}$ Que la tribu des Gryllotalpiens forme une chaîne qui se rattache par un bout aux Gryllides par les Gryllotalpa, et par l'autre aux Acridides, et spécialement aux Tetticides, par les Rhipipteryx; les Tridactylus formant le terme intermédiaire ${ }^{1}$.

$2^{\circ}$ Que la tribu des Gryllotalpiens ne peut être caractérisée que d'une manière très générale, et comme șuit :

Abdomen tantôt simple, dépourvu d'oviscapte chez les femelles, tantôt muni d'un oviscapte court du genre de celui des Acridides. Tibias antérieurs très comprimés, plus ou moins fortement dilatés et armés de griffes digitales formant un instrunent aratoire. Tarses composés de 3,2 ou 1 article seulement.

Les Tridactylites offrent des caractères si particuliers qu'il convient d'en former une sous-tribu séparée. La tribu se divisera comme suit :

\section{Sous-tribu des Gryllotalpites.}

Élytres (lorsqu'il existe des organes du vol) raccourcis, membraneux, oftrant chez les mâles un tambour. Ailes membraneuses, conformes à celles des Grylliens.

Pattes robustes. La paire antérieure très dilatée; ses tibias très comprimés et très larges, armés de 2-5 doigts robustes;

' Comme l'a indique L.-H. Fischer, le tube intestinal des Tridactylus offre des analogies avec celui des Acridides; il ressemble plus a celui de ces insectes qu'à celui des Gryllotalpites. 
ses tarses terminés par deux griffes droites (ou nulles). Pattes postérieures courtes et faibles, peu sauteuses ou ne l'étant pas du tout. - Abdomen simple, sans distinction dans les deux sexes; le segment anal muni des deux appendices ordinaires seulement (cerci).

a. Antennes longues et sétacées. Tarses tous composés de 3 articles; ceux des $2^{\text {me }}$ et $3^{\text {me }}$ paires munis de griffes ordinaires à deux crochets. Tibias postérieurs terminés par 6 éperons. Corps ailé. Gryllotalpa. - Scapteriscus.

$a, a$. Antennes très courtes, monoliformes, composées d'un petit nombre d'articles. Tarses composés de 1 ou 2 articles, à griffe unique. Tibias postérieurs armés de 4 éperons seulement. Corps aptère. - Cylindrodes.

\section{Sous-tribu des TridactYlites.}

Insectes ailés. Élytres raccourcis et cornés, dépourvus de tambour chez les mâles. Ailes flabelliformes, à champ marginal corné. Pattes très comprimées. Tibias antérieurs plus ou moins dilatés, parcourus à leur face interne par une rainure servant à recevoir le tarse. Tarses des deux premières paires grêles, composés de deux articles et munis de griffes ordinaires à deux crochets. - Pattes postérieures très fortement sauteuses; leurs fémurs monstrueux, énormément dilatés; leurs tibias très grêles, terminés par 4 éperons, dont les deux inférieurs très longs; leur tarse réduit au seul métatarse (ou nul).

Abdomen dépourvu d'oviscapte ou muni, chez les femelles. d'un oviscapte court. (Plaque suranale étroite et prolongée). Segment anal muni de 4 appendices anaux. Antennes courtes, composées d'un petit nombre d'articles distincts. - G. Tridactylus. - Rhipipteryx.

Obs. On remarquera que les Cylindrodes et les Tridactylites, malgré l'extrême divergence de leur genre de vie, possèdent des caractères communs, soit: dans la brièveté des antennes, dans le nombre réduit des éperons de leurs tibias postérieurs et des 
articles de tous leurs tarses. Ces modifications revêtent toutefois, dans ces deux types, un caractère fort différent, dépendant d'adaptations absolument différentes et n'indiquent aucune affinité.

\section{APPENDICE}

\section{Sur le genre Cylindrodes.}

Ce singulier genre étant encore très peu connu, nous en donnons la description, dans la limite du possible, d'après deux individus, malheureusement fort détériorés, qui sont en notre possession.

Les Cylindrodes pourraient être envisagés comme des Gryllotalpa qui auraient été passées à la filière, de manière à s'être considérablement rétrécies et allongées, tout en prenant une forme cylindrique et à peu près égale partout. Cette forme est une conséquence forcée du genre de vie de ces insectes, qui progressent dans l'intérieur des grandes plantes herbacées en s'y creusant des canaux étroits, juste suffisants pour leur donner passage.

Il faut supposer qu'ils opèrent ce travail en dévorant la moelle ou la substance de ces plantes en s'aidant de leurs pattes antérieures pour élargir la galerie d'avancement au point de donner passage au prothorax. Le reste du corps devant passer par le même vide, il a fallu que l'abdomen se rétrécisse au même calibre que le prothorax et que les pattes des $2^{\text {me }}$ et $3^{\text {me }}$ paires pussent s'effacer complètement, afin de ne pas augmenter le volume du thorax.

Description. La surface du corps est partout cornée et fortement cuirassée, à l'abdomen presque comme au thorax, lisse, non veloutée comme chez les Gryllotalpa, mais toutes ses pièces sont frangées de poils sur leurs bords.

La tête est conique comme chez les Gryllotalpa et ne porte 
non plus que deux ocelles, mais ceux-ci ne sont pas développés et n'apparaissent que sous la forme de deux taches jaunes. Les yeux sont très écartés, petits et point saillants. L'écusson facial est soudé au front, et sa surface est unie.

La carapace du pronotum est entièrement cylindrique, rejetant en avant les pattes antérieures et enveloppant le corps; ses deux bords latéraux se rejoignant en dessous, suivant une suture longitudinale. Le mésothorax forme un cylindre complet; sa partie dorsale est divisée en longueur par deux sillons, qui le partagent en trois compartiments, dont le médian représente le tergite et les deux latéraux, les pleurites (épisternites) très dilatés, refoulés au sommet des flancs par le mésosternum, parce que celui-ci forme un demi-cylindre complet, occupant toute la moitié inférieure du mésothorax et remontant sur les côtés jusqu'au milieu des flancs. Les pièces dorsale, latérales et sternale sont soudées les unes aux autres, ne laissant entre elles aucun vide, et formant une cuirasse cylindrique. (Chez les Gryllotalpa, le mésosternum est comprimé et caréné au milieu, et ne remonte pas sur les côtés.) Les côtés du mésothorax sont en dessus dans leur partie postérieure, fortement excavés pour faire place à la base des fémurs; il résulte de là que le lobe dorsal médian (tergite) se termine en arrière en forme de biseau. Le métanotum et les deux premiers tergites de l'abdomen sont, eux aussi, entièrement excavés, ou pour le moins obliquement aplatis sur les côtés, pour faire place aux pattes intermédiaires et postérieures, repliées, et leur permettre de s'effacer au point de ne pas augmenter la largeur du corps. Il en résulte que ces segments n'ont presque plus aucune largeur en dessus, et qu'ils sont comme comprimés en forme de lame ou de mur. Le métanotum est très court; il se dissimule entre les genoux des pattes intermédiaires, et sa crête est faiblement échancrée Le tergite du segment médiaire, plus long, n'est lamellaire qu'à sa base et s'élargit en arrière en entonnoir; d'autres fois, il est 
entièrement comprimé, ainsi que la base du $2^{\text {me }}$ tergite de l'abdomen, et c'est alors ce dernier seulement qui s'élargit en triangle à son extrémité postérieure. Le métasternum est plat, de forme trapézoïdale, et parcouru par deux sillons un peu convergents en arrière; il ne remonte pas sur les côtés, aussi les pleurites occupent-ils presque leur position normale en avant des hanches, formant le bas des flancs.

Tous les segments de l'abdomen se composent de 4 plaques cornées : le tergite cylindrique, le ventrite plus aplati et moins large, et deux pièces latérales étroites et parallèles (pleurites) un peu infères, qui unissent les plaques dorsales et ventrales suivant des sutures membraneuses et mobiles. L'abdomen est beaucoup plus long que le thorax; il s'est allongé en proportion de son rétrécissement. Ses segments ne sont pas aussi transversaux que chez les Gryllotalpa; le $4^{\text {me }}$ est aussi long que large; les suivants vont se raccourcissant un peu jusqu'au $8^{\text {me }}$. L'extrémité de l'abdomen diffère notablement de celle des Gryllotalpa ${ }^{1}$. Le $9^{\text {me }}$ tergite est très grand, aussi long que le $4^{\mathrm{me}}$, voûté et convexe, un peu rabattu sur les côtés, et arrondi en arrière. Le $10^{\text {me }}$ est peu apparent, très court, ne formant qu'une étroite bande, presque linéaire, difficile à distinguer et rabattue en bas, offrant une dépression médiane; mais il se prolonge au milieu en forme de dé à coudre, pour former la plaque suranale (l'anus étant mal conservé chez notre individu, cette description est un peu incertaine).

Le dernier segment ventral ( $9^{\text {me }}$ ventrite) est grand, arrondi en arrière. Les pleurites du $8^{m}$ segment sont peu coriacés. Le $10^{\text {me }}$ segment offre de chaque côté une plaque cornée. Les cerci s'articulent au sommet de ces plaques; ils sont très courts, cylindriques et brièvement subulés, non divisés en articles.

Pattes. Les pattes sont toutes très courtes, très comprimées

${ }^{1}$ Voir p. 406 et fig. 17-19. 
et très appliquées au corps, ne faisant nullement saillie sur les côtés. A cet effet, au lieu d'être insérées latéralement, elles sont rejetées à l'extrémité des segments qui les portent et s'insèrent, les antérieures au bord antérieur du prothorax, les autres au bord postérieur des méso- et métasternum. Elles n'augmentent en rien l'épaisseur du cylindre du corps. Au contraire, le corps est fortement rétréci entre le mésothorax et le $3^{\text {me }}$ segment de l'abdomen, afin de laisser un peu de mobilité aux deux paires de pattes postérieures, lors même que le calibre de la galerie que perce l'insecte n'est déterminée que par la grosseur du prothorax.

Les pattes antérieures sont fortement dilatées. Le fémur est carré-arrondi et n'a presque aucune épaisseur. Sa face externe est à peine convexe, fortement ponctuée, luisante et rugulée. Sa base arrondie chevauche en dehors par-dessus l'extrémité de la hanche; son bord supérieur est caréné, et son extrémité a l'air d'être formée par des écailles imbriquées, mais cette apparence tient seulement à ce qu'elle est divisée en compartiments par des carènes. On voit d'abord une carène transversale partant de l'extrémité du bord supérieur et se prolongeant en arrière au-dessus du bord inférieur, séparant la partie apicale du fémur. Celle-ci est lamellaire et fendue, formant deux lobes arrondis, dont l'inférieur est le plus grand et lui-même partagé par une carène arquée. Le trochanter, rejeté en dessous, longe le fémur, dont il n'est séparé que par un sillon, et forme avec le fémur un seul tout. Il est partagé par une carène longitudinale; sa moitié supérieure est de forme lancéolée et subcannelée; sa partie inférieure est de moitié plus courte. Le bord du lobe apical inférieur du fémur se continue en une seule courbe avec la carène du trochanter; il est faiblement courbé en dehors et tranchant à la manière d'une truelle.

Le tibia est lamellaire, arqué en arc de cercle et découpé à son bord supérieur de manière à former 5 dents légèrement courbées en dehors. Il s'articule fortement au bord supérieur 
du fémur dans le lobe supérieur de ce dernier, et peut se replier sous le lobe inférieur et l'extrémité du trochanter, de façon à ce que tout ou partie de ses dents dépassent le bord apical du fémur.

Le fémur, le trochanter et le tibia forment ainsi, par leur ensemble, comme un soc de charrue, capable de rejeter en dehors la moelle des plantes dans lesquelles travaille l'insecte.

Le tarse n'ajoute rien à cet ensemble. Il est réduit à un seul article allongé, grêle et cylindrique, dépourvu de griffe, cilié en dessous et terminé par quelques soies; il s'articule entre les deux dernières dents du tibia, et s'efface en se repliant contre la face interne de ce dernier. Il ne semble exister que par réminiscence.

Les fémurs sont fortement ciliés sur leurs bords, et les tibias portent également quelques poils dans leurs échancrures.

Les pattes intermédiaires et postérieures sont presque similaires; très courtes, fortes, ciliées. Les fémurs sont lenticulaires, comprimés, à bord supérieur très arqué. Les tibias sont dépourvus d'épines; ceux de la paire intermédiaire ont leur bord apical-antérieur oblique et découpé de deux échancrures dessinant 3 dents, dont la première obsolète, la $2^{\text {me }}$ triangulaire, la $3^{\text {me }}$ dentiforme. Il y a en outre souvent une $4^{\mathrm{me}}$ dent, formant la paire avec la $3^{\mathrm{me}}$, mais ces dents sont sujettes à l'atrophie (elles manquent presque complètement à la patte droite d'un de nos individus). Les tibias postérieurs se terminent par 4 dents bien développées, soit par 4 éperons, 2 supérieurs, 2 inférieurs. Les tarses se composent de deux articles et sont armés d'une griffe unique, assez longue, presque droite et aiguë.

Les hanches intermédiaires sont très courtes et très écartées afin de permettre aux genoux de converger sur le dos (comme le font ceux des pattes postérieures dans tous les Gryllotalpites). Il en résulte que le mésosternum est plus large que le métasternum.

Les pattes postérieures ne sont pas plus grandes que les in- 
termédiaires; elles sont même un peu moins fortes, mais le fémur est un peu moins court. Ces pattes sont absolument inaptes au saut.

Les pattes intermédiaires et postérieures n'ont d'autres fonctions que de fixer le corps dans les galeries au moyen des dents apicales de leurs tibias et de le chasser en avant, tandis que les pattes antérieures travaillent à avancer la galerie; elles servent aussi à faire progresser l'insecte dans des canaux déjà percés. Lorsque l'insecte abandonne son réduit pour passer d'une plante à une autre, elles deviennent ambulatoires. Les pattes antérieures deviennent, elles aussi, ambulatoires, grâce à leurs tibias, qui, repliés contre le fémur pour le travail aratoire, peuvent se dégager et appuyer sur le sol par leur extrémité.

Toutes les pattes étant, chez les Cylindrodes, beaucoup plus courtes et beaucoup plus appliquées que chez les Gryllotalpa, les mouvements dont elles sont susceptibles sont beaucoup moins amples que chez ces dernières; les pattes antérieures en particulier ne fournissent qu'un très petit mouvement latéral. Les Cylindrodes ne peuvent, à cause de cela, percer que des galeries très étroites, et non, comme les Gryllotalpa, des chemins assez larges pour s'y mouvoir avec rapidité. Il faut dire que le travail des Cylindrodes dans les tiges des plantes est beaucoup plus ardu que celui des Gryllotalpa dans le sol, la terre étant bien plus facile à percer et à déplacer que la substance du tissu végétal, aussi les Cylindrodes sont-ils forcés de réduire leur travail au minimum possible et de se contenter de percer des galeries juste assez larges pour donner passage à leur corps.

Les Cylindrodes offrent dans toutes les parties de leurs corps des modifications surprenantes qui, par leur ensemble, constituent un merveilleux exemple d'adaptation à un genre de vie sui generis, et qui va jusqu'à détruire une partie des caractères de famille (pattes postérieures non sauteuses et sans épi- 
nes; griffes simples), et de tribu (antennes très courtes; articles des tarses réduits) ${ }^{1}$.

\section{Espèces.}

GRAY a établi le genre Cylindrodes d'après une espèce qu'il a nommée Campbelli. Nos individus ne correspondent pas exactement à la description qu'il en donne. Nous distinguerons les trois formes suivantes ?

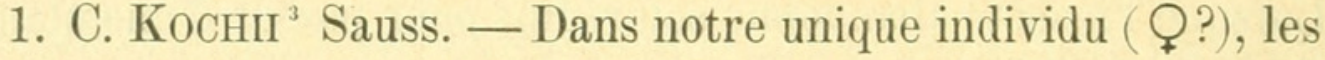
deux premiers tergites de l'abdomen et la base du $3^{\text {me }}$ sont comprimés d'une manière lamellaire. Les pans latéraux de ces segments sont fortement excavés sur les côtés, même au $3^{\text {me }}$ tergite de l'abdomen, en sorte que les fossettes latérales se prolongent jusqu'à l'extrémité de ce segment et qu'elles servent à loger les tibias postérieurs, lors même que ceux-ci ne sont pas repliés contre les fémurs. La crête dorsale est tout à fait lamellaire au métanotum; aux deux segments suivants, elle l'est un peu moins, et arrondie en dessus. Le $3^{\text {me }}$ tergite s'élargit d'avant en arrière en triangle un peu arrondi en dessus; son extrémité se perd dans un plan oblique plus large et divisé en 3 compartiments excavés et polis, dont les deux latéraux sont triangulaires, le médian plus grand, en triangle renversé, à bord supérieur arqué. Ce compartiment est lui-même divisé en 9 fossettes

${ }^{1}$ Parmi ces Hyménoptères du genre Scolia, on trouve des différences de mœurs presque parallèles à celles des Gryllotalpites. La plupart des Scolia s'enfoncent dans la profondeur du sol en remuant la terre, mais la $S c$. oryctophaga, et sans doute d'autres espèces aussi, pénètre dans les galeries que les Oryctes creusent dans les troncs des arbres. Toutefois, de cette différence dans le genre de vie, il ne rèsulte pas chez cette Scolie de modifications morphologiques, attendu que l'insecte ne perfore pas le bois des arbres, mais ne fait que profiter des galeries déja établies par les Coléoptères dont il est le parasite en se frayant un chemin dans le terreau qui remplit ces excavations. (Cp. H. de Saussure. Ap. Grandidier, Histoire de Madagascar, t. XX, Hyménoptères, p. 18ə̈, notes.)

${ }^{2}$ Pour la description des espèces, voir aussi H. De Saussure, Mélanges orthopterol., Ve fascic., p. 40.

${ }^{3}$ H. De Saussure, Mélanges orthoptérol., כ̈me fasc., pl. 11, fig. III. 
dont les deux latérales forment des cannelures obliques. Toutes ces fossettes sont séparées par des arêtes ${ }^{1}$. Le $4^{\mathrm{m} \theta}$ tergite est séparé du $3^{\mathrm{me}}$ par un large sillon ou par une sorte d'étranglement bordé par quelques petites impressions. Au $3^{\text {me }}$ tergite, les cavités latérales sont bordées supérieurement par des arêtes arrondies, qui deviennent vives à l'extrémité du tergite. Les fémurs des $2^{\mathrm{me}}$ et $3^{\mathrm{me}}$ paires sont absolument mutiques. Leur bord inférieur se termine à la face externe par un arrondissement qui, par suite d'une petite échancrure du bord apical, forme presque un lobe géniculaire arrondi.

Le dernier tergite de l'abdomen ne porte pas de sillon, et le dernier ventrite est plus court que chez le $\mathrm{n}^{0} 2$.

2. Species...? (Q?). - Chez cet individu, les deux premiers tergites seulement de l'abdomen sont comprimés, mais non d'une manière lamellaire; ils offrent en dessus un replat étroit, qui s'élargit en arrière. Le premier est lamellaire à son extrême base et forme ensuite un dos d'âne arrondi; le $2^{\text {me }}$ offre en dessus un replat en forme d'entonnoir étroit, un peu tronqué à sa base et plus aplati que le replat du premier; le $3^{\text {me }}$ tergite est presque cylindrique. Les côtés du métanotum et de ces tergites ne sont guère excavés, mais seulement obliquement aplatis, et l'aplatissement se continue en mourant sur les côtés du $3^{\text {me }}$. Le bord supérieur des facettes ainsi formées est arrondi et n'est plus appréciable au $3^{\mathrm{me}}$ tergite. Les fémurs des $2^{\mathrm{me}}$ et $3^{\mathrm{me}}$ paires sont mutiques, mais le bord inférieur-externe se termine à la paire du milieu par un lobe géniculaire triangulaire, qui est formé par une échancrure triangulaire du bord apical. Le $9^{\text {me }}$ tergite (fig. 17) est grand, partagé avant le milieu par un sillon transversal incomplet. Les cerci sont très apparents; le $9^{\text {me }}$ ventrite (fig. 18) est assez allongé.

3. C. Campbelli Gray. - Cette espèce a été figurée par

${ }^{1}$ Il n'est guère à supposer que cette sculpture si régulière soit le résultat d'une déformation. 
Gray (Griffith, Animal Kingdom, t. XV, Ins. p. 785, Pl. 131, sexus?). Notre $n^{0} 2$ correspond parfaitement à la figure de GraY, si ce n'est que sur cette dernière le $9^{\text {me }}$ segment de l'abdomen est plus grand et quelque peu dilaté. Mais l'auteur dit que les fémurs des $2^{\text {me }}$ et $3^{\text {me }}$ paires se terminent par une dent qui sert à ces insectes à se fixer contre les parois des galeries dans lesquelles ils se meuvent '.

L'auteur donne au tarse antérieur deux articles, et les cerci ne seraient pas apparents?

Le genre de vie des Cylindrodes permet de supposer qu'il en existe plusieurs espèces, habitant chacune une espèce ou un groupe d'espèces de plantes.

${ }^{1}$ Les Gryllotalpiens n'offrent pas d'épines géniculaires. L'auteur n'aurait-il pas fait un lapsus calami et écrit fémurs au lieu de tibias? S'il en est ainsi, le $\mathrm{n}^{\circ} 2$ rentrerait dans le $C$. Campbelli. 


\section{EXPLICATION DES PLANCHES}

LÉGEnde: 1-10, segments de l'abdomen suivant leur numéro d'ordre. $9 i$ neuvième segment ventral (ou plaque sous-génitale, chez le mâle). $-z$ dixième tergite. - vs son prolongement formant la valvule superieure de l'anus ou plaque suranale. - vi valvules inférieures de l'anus. - c cerci. - $a$ appendices anaux. - $8 u$ valves inférieures de l'oviscapte. - 90 valves supérieures de l'oviscapte. 
Fig. 1-7. Gryllotalpa vulgaris L.

Fig. 1. L'extrémité de l'abdomen 오, face ventrale.

Fig. 2. , 0 ,

Fig. 3. Le pénis du mâle, vu en dessus. - $p$ sa partie charnue. $f$ fossette de sa base. - $g$ sillon longitudinal. - $b$ branche transverse de l'ancre corné. - $s, r$ les deux cornes latérales de cette branche (Cp. fig. 4, 5). - $i$ l'extrémité de la branche longitudinale de l'ancre. $-t$ les titillateurs (cp, fig. 6).

Fig. 4. L'ancre cornée du pénis vue en dessus. - $b$ sa branche transverse. - $r, s$ ses cornes terminales. - o sa branche longitudinale. Cette branche étant courbée, elle n'apparaît sur la fig. 3 (i) qu'en fort raccourci.

Fig. รั. La même vue en dessous.

Fig. 6. Les titillateurs vus en dessus. $-c$ leur partie postérieure dilatée en cuilleron. - $e$ leur partie antérieure engagee sous la branche longitudinale de l'ancre. (Sur la fig. 3 on ne voit que leur partie postérieure $c$ ).

Fig. 7. Un titillateur vu de profil.

Fig. 8-14. Gryllotalpa africana, P.-B.

Fig. 8. L'extrémité de l'abdomen $\sigma^{\pi}$, face dorsale. $-z$ dixième tergite. vs valvule supérieure de l'anus ou plaque suranale. - vi valvules inférieures de l'anus. $-c$ cerci.

Fig. 9. L'extrémité de l'abdomen $\sigma^{x}$ vue par derrière el ouverte. - Lettres comme pour la fig. 8 . $-n$ anus. $-m$ membrane sous-anale (correspondant peut-être au $10 \mathrm{me}$ tergite). - $8 i$ huitième segment ventral déplié. - $f$ neuvième segment ventral ou plaque sous-génitale. - $p$ partie charnue du pénis.

Fig. 10. Le pénis vu en dessus. -- $p$ partie charnue. $-b$ ancre corné. $o$ sa branche longitudinale. $-v$ corps charnus.

Fig. 11. Le même vu en dessous.

Fig. 12. Le même vu de profil.

Fig. 13. Les titillateurs, ici soudés en une seule pièce. ( $c p$ fig. 6.)

Fig. 14. Le titillateur droit vu de profil.

Fig. 15, 16. Scapteriscus didactylus L.

Fig. 15. Lancre cornée du pénis (notations comme pour les fig. 4, כ̆).

Fig. 16. Un titillateur.

Fig. 17-19. Genre Cylindrodes (ㅇ ?)

Fig. 17. L'extrémité de l'abdomen, face dorsale.

Fig. 18. La même, face ventrale. - z le $10 \mathrm{me}$ tergite très peu apparent, prolongé au milieu pour former la plaque suranale.

Fig. 19. L'extrémité de l'abdomen, face ventrale, dont on a enlevé les $8^{\text {me }}$ et $9 \mathrm{me}$ ventrites. - vs valvule supérieure de l'anus (plaque suranale). - $b$ bourrelet charnu terminé par deux pointes cornées.

Fig. 20-24. Tridactylus variegatus.

Fig. 20. Les deux derniers segments ventraux de l'abdomen ( 8 me et 9 me) vus en dessous. + . $-u$ annexe du 8 me ventrite (fendu). $-o$ le dernier segment partagé par un sillon.

Fig. 21. L'extrémité de l'abdomen, soit le segment anal (10me segment) $\mathrm{vu}$ en dessus. $-z$ dixième tergite prolongé au milieu entre le cerci pour former la plaque suranale et comprimé. - $c$ cerci. $a$ appendices anaux.

Fig. 22. Id. vue de profil. - vi valvule anale inférieure droite. $-x$ plaque chitinisèe.

Fig. 23. Id. $\sigma^{\mathbb{T}}$ vue en dessous, après l'enlèvement de la plaque sous-génitale (9me ventrite) pour montrer que les appendices anaux inférieurs $a$ ne sont que des prolongements des valvules inférieures de l'anus vi.

Fig. 24. Id. vue en dessus. - vs plaque suranale. 


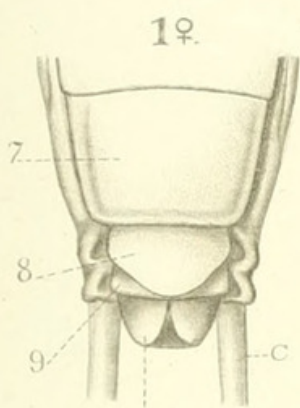

$8 \sigma^{\circ}$
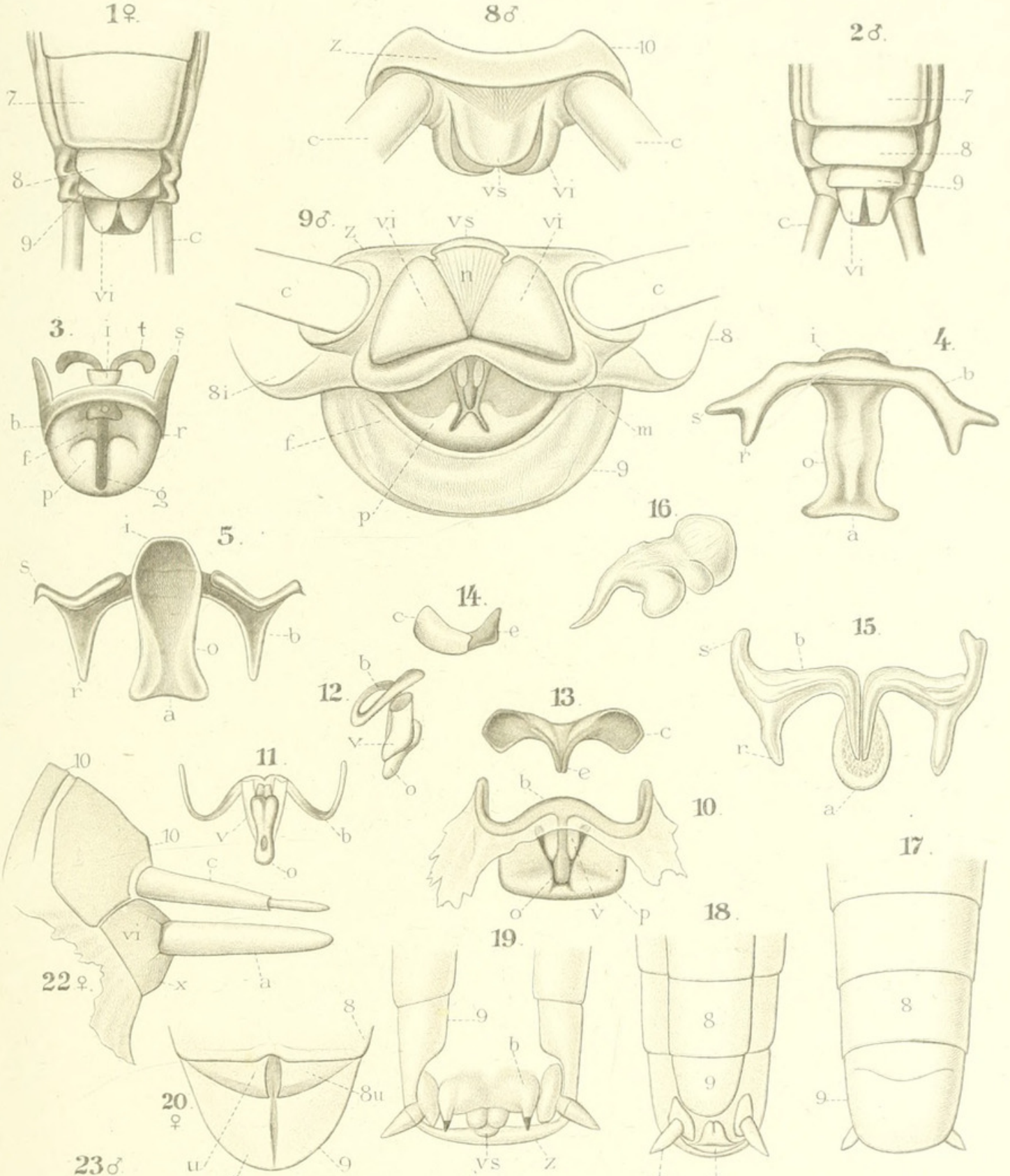

23 ช

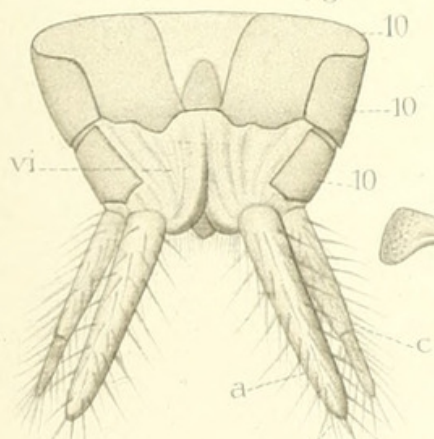

10

6.
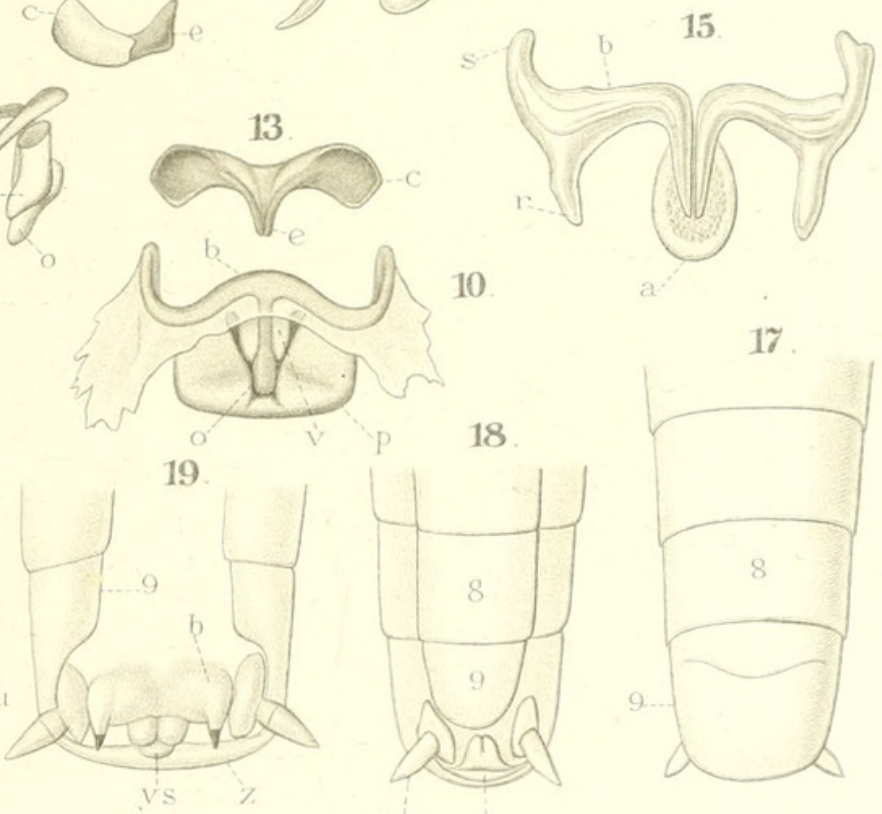

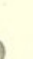

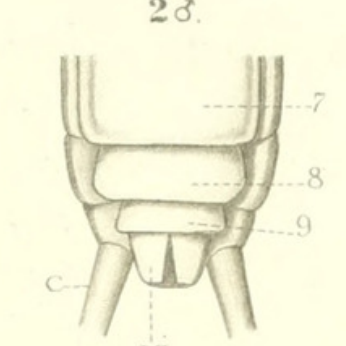


Fig. 20̈-34. Rhipipteryx mexicana Sss.

Fig. 2\%. L'extrémité de l'abdomen $q$, face dorsale. $-c$ cerci. $-a$ appendices anaux. $-8 u$ valves inférieures de l'oviscapte. -90 valves supérieures de l'oviscapte.

Fig. 26. Id. face ventrale (mêmes notations que pour la fig. 2כ).

Fig. 27. Id. de profil. - $8 i$ plaque sous-génitale dejpendant du 8 me sternite.

Fig. 28. L'extrémitè de l'abdomen $\sigma^{x}$, face dorsale. - vs plaque suranale avec ses annexes latéraux. $-9 i$ plaque sous-génitale (9me ventrite). - * plaques chitinisées.

Fig. 29. La mème, face ventrale.

Fig. 30. La même de profil.

Fig. 31. Le segnent anal $\$$ (10me segment) vu en dessus, ouvert, déplié et un peu déprimé. - $l$ parties latérales du 10 me tergite $(=z$, fig. 8, 21, 24). - $r$ arc corné unissant les parties latérales. vs plaque suranale (valvule anale supérieure). - vi valvules anales inférieures portant les appendices anaux $a$. $-i$ tube intestinal.

Fig. 32. Id. vu en dessous, disséqué pour montrer le tube intestinal $i$. L'arceau corné $r$ est vu par transparence à travers le rectum.

Fig. 33. Id. vu en dessous, le 9me segment ventral étant enlevé.

Fig. 34. Le segment anal $\sigma^{x}$ vu par derrière, ouvert et fortement comprimé (déformé), pour montrer les plaques cornées de la membrane qui unit les différentes parties du segment — * plaque en forme de crochet.

Fig. 33̆, 36. Rhipipteryx cyanipennis Sss. $\sigma^{7}$.

Fig. 3כ̃. L'extrémité de l'abdomen vue par sa face dorsale. - $9 s$ neuvième tergite déplié et relevé, vu par son bord supérieur (soit son bord postérieur relevé). - $9 i$ plaque sous-génitale.

Fig. 36. Id. vue de profil.

Fig. 37. L'extrémité de l'abdomen du Rh. forceps Sss. $\sigma^{*}$. - vs plaque suranale, ou valvule supérieure de l'anus. - $9 i$ plaque sous-génitale. $-c$ cerci. $-a$ appendices anaux. 
25 우
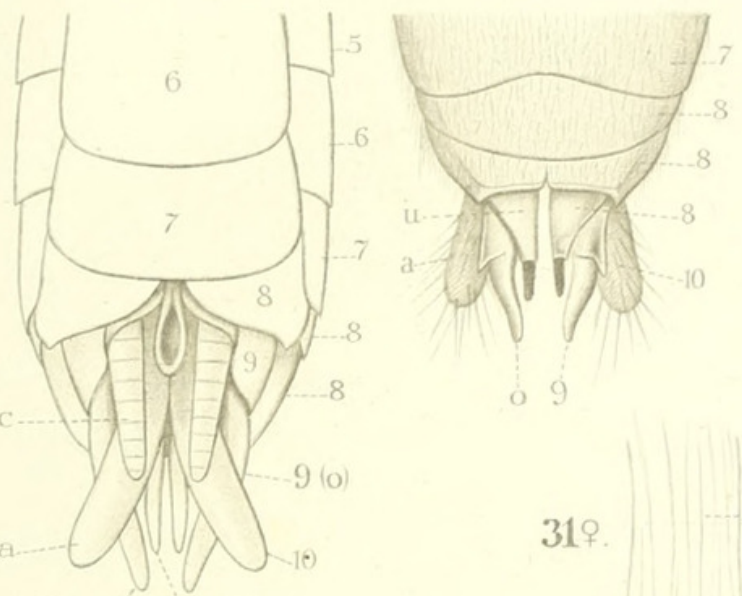

o 9

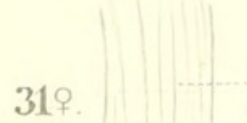

$29 \sigma^{\circ}$

$280^{\circ}$

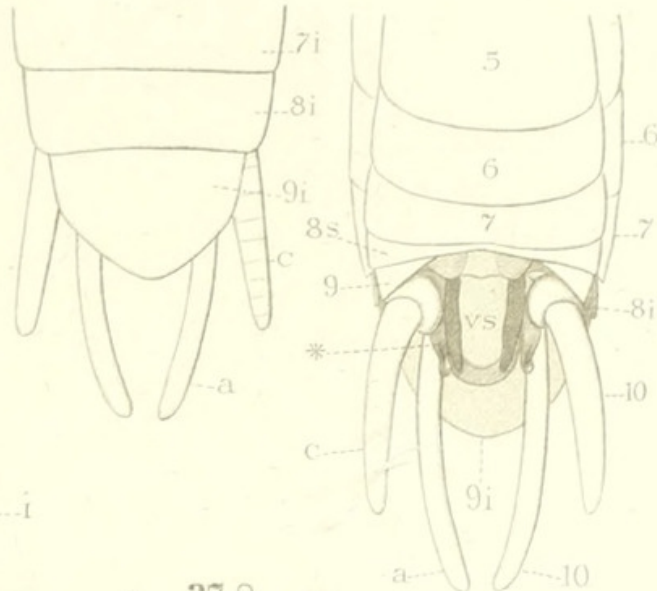

27 우

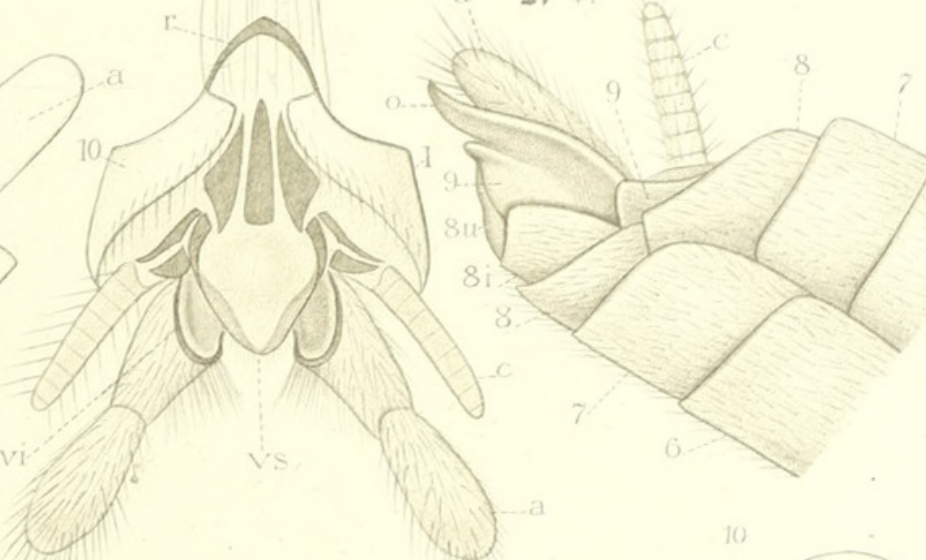

30 б
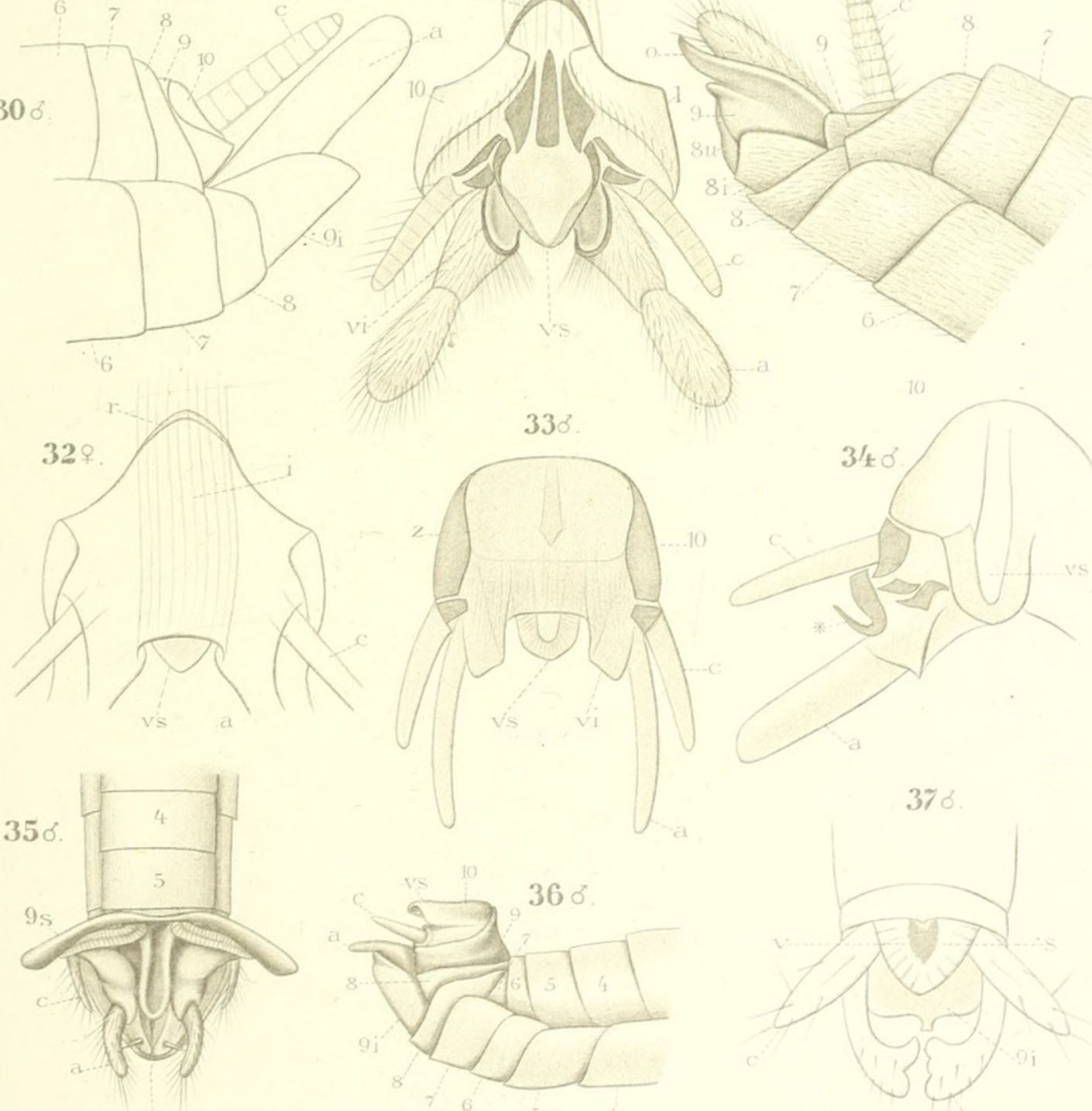

37 ช.

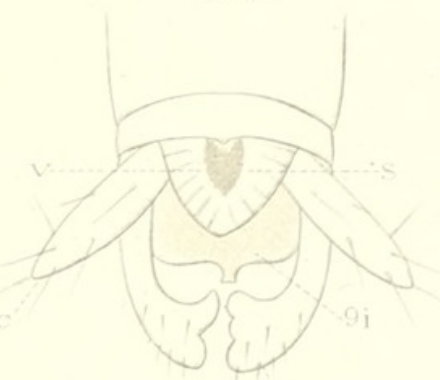

Zehntner del

de Saussure \& Zehntner . Gryllotalpiens 


\section{$2 \mathrm{BHL}$ Biodiversity Heritage Library}

Saussure, Henri de and Zehntner, Leo. 1894. "Notice morphologique sur les Gryllotalpiens." Revue suisse de zoologie 2(3), 403-430.

https://doi.org/10.5962/p.318228.

View This Item Online: https://www.biodiversitylibrary.org/item/38139

DOI: https://doi.org/10.5962/p.318228

Permalink: https://www.biodiversitylibrary.org/partpdf/318228

\section{Holding Institution}

MBLWHOI Library

Sponsored by

MBLWHOI Library

\section{Copyright \& Reuse}

Copyright Status: NOT_IN_COPYRIGHT

This document was created from content at the Biodiversity Heritage Library, the world's largest open access digital library for biodiversity literature and archives. Visit BHL at https://www.biodiversitylibrary.org. 